\title{
AN ISOTOPIC BIOGEOCHEMICAL STUDY OF THE OXFORD CLAY FORMATION (U.K.)
}

\author{
KENIG*, Fabien, and HAYES, John M., Biogeochemical Laboratories, Geology Bldg., \\ Indiana University, Bloomington, IN 47405, U.S.A.; SUMMONS, Roger, Bureau of \\ Mineral Resources, P.O. Box 378, Camberra, 2601, AUSTRALIA.
}

Lipids from a suite of 12 representative samples from the Lower Oxford Clay (LOC) and from the Middle and Upper Oxford Clay (MUOC) were extracted with organic solvent, separated, and analyzed qualitatively and quantitatively using gas chromatography-mass spectrometry (GC-MS) and isotopically using isotope-ratio-monitoring gas chromatography-mass spectrometry (irmGCMS). Determination of molecular structures allowed identification of compounds which retained enough chemical structural information to be recognizable as having a biochemical origin related to a specific organism or group of organisms (biomarkers). By allowing assignment of each compound to a portion of the local carbon cycle (production, sedimentation, and alteration), isotopic analyses of individual compounds provided further information regarding their origins. The 12 samples were chosen to represent the various macrofaunal assemblages define by Duff (1975) and to cover the total range of variability of the bulk geochemical parameters measured on sediments from LOC and MUOC (Kenig et al., this symposium).

A relatively high content of unsaturated hydrocarbons, as well as the abundance of biogenic forms of molecular stereoisomers such as $\beta \beta$ hopanes and $\alpha \alpha \alpha$ steranes and other molecular indicators, confirms the low level of maturity of organic matter in the LOC. Contributions from the two main sources of sedimentary organic matter already identified from bulk geochemical data, marine phytoplankton and terrestrial organic matter, can be recognized in the saturated hydrocarbon fraction of all the samples analyzed. However, contributions from these two sources vary as a function of the TOC content, the HI, and the $\delta^{13} \mathrm{C}$ of organic matter. Saturated hydrocarbon fractions of high-TOC samples, formed during periods of high marine productivity, are mainly dominated by pristane and phytane and by tetra- and pentacyclic biomarkers. This dominance decreases with the TOC content and the ${ }^{13} \mathrm{C}$ abundance. Conversely, the relative abundance of compounds indicative of terrigenous input increases. In samples with lower TOC, $n$-alkanes with a strong odd-carbon preference, peaking at $C_{27}$, are particularly prominent in the saturated hydrocarbon fraction.

Biosynthetic products with $n$-alkyl carbon skeletons are found in nearly all organisms. Accordingly, sedimentary $n$-alkanes are likely to derive from multiple sources. These can be partly distinguished by isotopic analysis of individual $n$-alkanes. In particular, marine and terrestrial sources can be resolved. Considering the variation of their relative abondance and of their isotopic composition as a function of TOC, $\delta^{13} \mathrm{C}$ of total organic matter and hydrogen index, the origins of $n$-alkanes, pristane and phytane as well as those of other biomarkers will be discussed. The paleobiological assemblage responsible for the production and alteration of the organic matter will be tentatively reconstructed. 